Rev. Inst. Flor. v. 28 n. 1. p. 59-67 jun. 2016

http://dx.doi.org/10.4322/rif.2016.004

ISSN impresso 0103-2674/on-line 2178-5031

\title{
O PAPEL DAS AVES NA DISPERSÃO E GERMINAÇÃO DE SEMENTES DO PAU-INCENSO (Pittosporum undulatum Vent.) EM UM REMANESCENTE DE MATA ATLÂNTICA ${ }^{1}$
}

\author{
BIRD ROLE IN SEED DISPERSION AND GERMINATION \\ OF Pittosporum undulatum Vent. SEEDS IN AN ATLANTIC FOREST REMNANT
}

\author{
Mariana Lopes CAMPAGNOLI, 2,3; Sergio Roberto Garcia dos SANTOS²; \\ Sebastiana Dutra de Souza Revoredo da SILVA²; \\ Alexsander Zamorano ANTUNES ${ }^{2}$
}

\begin{abstract}
RESUMO - A invasão biológica pela espécie Pittosporum undulatum Vent. pode ocasionar degradação de ecossistemas nativos, perda de espécies e de serviços ecossistêmicos. Além das características biológicas que favorecem seu estabelecimento, seus frutos são fontes alimentares para algumas espécies de aves, que acabam por dispersar suas sementes. O presente estudo foi realizado no Parque Estadual Alberto Löfgren, e visou verificar as espécies de aves potencialmente dispersoras do pau-incenso, comparar os registros de alimentação obtidos para esta árvore e para outras seis espécies arbóreas locais e avaliar a influência da ingestão das sementes pelas aves na sua taxa de germinação. A coleta de dados consistiu em observações focais das aves em atividade de forrageio no pau-incenso e a captura de indivíduos com redes de neblina para coleta de sementes nas fezes. Testes de germinação foram realizados com as sementes coletadas das fezes e diretamente dos frutos maduros. No total, foram encontradas 107 sementes nas fezes de oito sabiás, pertencentes a três espécies: Turdus rufiventris, Turdus amaurochalinus e Turdus albicollis. Não encontramos diferenças significativas nas taxas de germinação entre os tratamentos. Apesar da passagem das sementes pelo trato digestório das aves não ter alterado significativamente as taxas de germinação, os sabiás podem ser importantes dispersores do pau-incenso, permitindo a colonização de novas áreas por esta espécie exótica e intensificando seu processo de invasão biológica na Mata Atlântica.
\end{abstract}

Palavras-chave: invasão biológica; dispersão; conservação; Turdus.

\begin{abstract}
Biological invasion by the species Pittosporum undulatum Vent. could bring degradation to native ecosystems and loss of biodiversity and ecological services. Besides the biological features favoring the establishment of the species in natural environments, some bird species use its fruits as food sources, being responsible for its seed dispersion. The present study was held at Alberto Löfgren State Park, São Paulo, Southeastern Brazil, and aimed to verify which bird species are $P$. undulatum potential dispersers; to compare feeding bouts to $P$. undulatum with another six arboreal species and to evaluate the influence of seed ingestion in germination rates. We sampled data using focal observation of foraging birds in $P$. undulatum and captured individuals with mist nets to collect seed from their faeces. In addition, we have made seed germination tests that included seeds collected from bird faeces and directly from mature fruits in trees. We found 107 seeds inside the faeces of eight thrushes, belonging to three species: Turdus rufiventris, Turdus amaurochalinus and Turdus albicollis. We did not find significant differences in germination rates between treatments. Although the passage through the birds' digestive system did not change significantly germination rates, thrushes can be important dispersers of P. undulatum, allowing colonization of new areas and intensifying the biological invasion process in Atlantic Forest.
\end{abstract}

Keywords: biological invasion; seed dispersion; conservation; Turdus.

\footnotetext{
${ }^{1}$ Recebido para análise em 01.03.2016. Aceito para publicação em 23.05.2016.

${ }^{2}$ Instituto Florestal, Rua do Horto, 931, 02377-000, São Paulo, SP, Brasil.

${ }^{3}$ Autora para correspondência: Mariana Lopes Campagnoli - marianacampagnoli@gmail.com
} 


\section{INTRODUÇÃO}

O pau-incenso Pittosporum undulatum Vent. é uma árvore originária da Austrália, disseminada ao redor do globo para uso paisagístico ou como cerca viva (Goodland e Healey, 1996). Em vários locais acabou se tornando uma espécie invasora, degradando os ecossistemas nativos, ocasionando a perda de espécies e de serviços ecossistêmicos. Mesmo em localidades com elevado número de espécies vegetais invasoras, o pau-incenso destaca-se por apresentar a maior densidade e frequência entre elas (Mielke et al., 2015). No Brasil, sua ocorrência como invasora foi assinalada principalmente em localidades florestais do Centro-Sul (Blum et al., 2005; Santana e Encinas, 2008; Instituto Hórus, 2016).

Pode ser considerada uma espécie oportunista, aproveitando-se de alterações ambientais resultantes da atividade humana para seu estabelecimento (Goodland e Healey, 1997). Entre as características que favorecem seu estabelecimento em uma região, podem ser citadas: elevada produção de propágulos (37.500 sementes por indivíduo), altos índices de germinação de sementes (Mullet, 1999a; 1999b), grande capacidade de adaptação a diferentes tipos de solo (Mullet, 1999b) e crescimento e rebrota favorecidos pela abertura de clareiras (Rose, 1997). Com isto, essa espécie ocupa o espaço disponível de maneira mais eficiente do que as espécies nativas e pode reduzir a diversidade local e dominar a comunidade vegetal (Rose, 1997).

A dispersão das sementes do pau-incenso pode ser hidrocórica ou zoocórica, sendo a ornitocoria a forma mais eficiente de disseminação (Cooper, 1959; Goodland e Healey, 1997; Mullet, 1999b; Blum et al., 2005; Heleno et al., 2011). No Brasil, os frutos do pau-incenso são reportados como fonte alimentar para sabiás Turdus spp. (Fonseca e Antunes, 2007). É possível que espécies da família Turdidae sejam potenciais dispersoras de suas sementes, uma vez que apresentam o comportamento alimentar de engolir os frutos inteiros (Moermond e Denslow, 1983; Parrini, 2015).
Alguns estudos demonstraram que a passagem de sementes pelo trato digestório de vertebrados, como as aves, pode influenciar nos padrões de germinação de algumas espécies vegetais (Barnea et al., 1991; D'Avila et al., 2010; Traveset e Verdú, 2002). A passagem da semente pelo trato digestório resulta no desgaste da polpa que a envolve, o que possivelmente aumenta sua permeabilidade à água e gases, consequentemente aumentando as taxas de germinação (Barnea et al., 1991; Traveset e Verdú, 2002).

Os objetivos do presente estudo foram verificar as espécies de aves potencialmente dispersoras do pau-incenso num remanescente de Mata Atlântica do Sudeste do Brasil, comparar os registros de alimentação obtidos para o pau-incenso com outras seis espécies arbóreas ornitocóricas locais e avaliar a influência da ingestão das sementes de pau-incenso na sua taxa de germinação.

\section{MATERIAL E MÉTODOS}

O estudo foi realizado no Parque Estadual Alberto Löfgren - PEAL (2327'41's e 46 37'54”W), zona norte da cidade de São Paulo. $\mathrm{O}$ parque possui altitude média de $776 \mathrm{~m}$, ocupa uma área de 174 ha e é contíguo ao Parque Estadual da Cantareira. Sua vegetação é constituída por talhões com plantios de espécies arbóreas nativas e exóticas, os chamados arboretos, e por trechos nos estádios de sucessão ecológica inicial e médio de Floresta Ombrófila Densa Montana. Algumas das espécies exóticas cultivadas, entre elas o pau-incenso, conseguiram se estabelecer naturalmente nos setores de vegetação nativa do parque (São Paulo, 2012).

No PEAL o plantio de pau-incenso ocorreu no arboreto da Vila Amália (Figura 1) em dois períodos distintos. Em 1926, no chamado lote 194, foram plantados indivíduos procedentes do Tahiti e, em 1939, no lote 160, mudas originárias da Argentina (João Batista Baitello, comunicação pessoal). Segundo o plano de manejo do PEAL, a disseminação natural dessa espécie em outras áreas do parque ocorreu pouco tempo após o seu plantio, estando, atualmente, distribuída de forma ampla nesta unidade de conservação (São Paulo, 2009). 


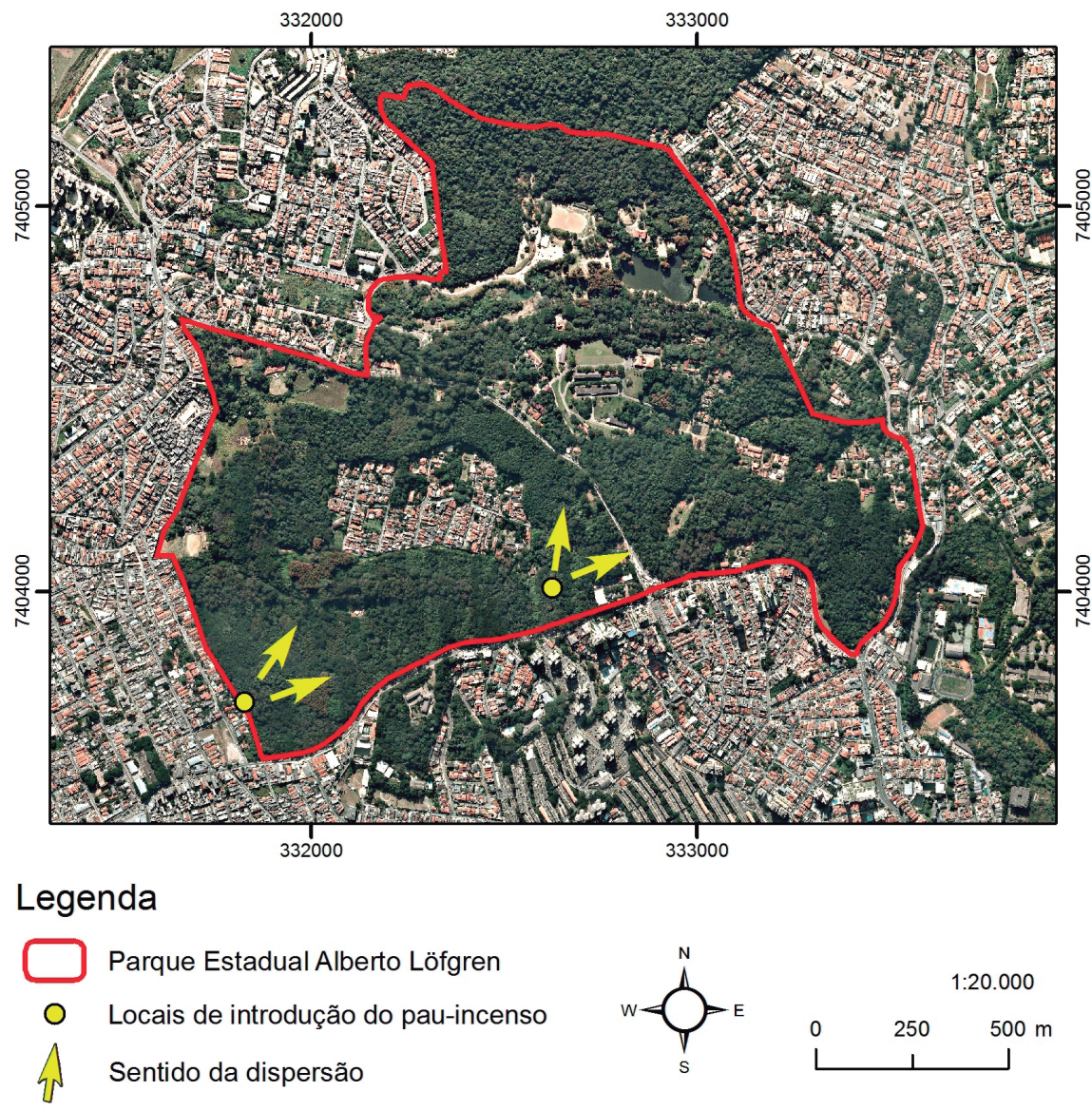

Figura 1. Área de estudo, indicando os locais de introdução de pau-incenso e o sentido da sua dispersão.

Figure 1. Study area, indicating Pittosporum undulatum introduction sites and the way of dispersion.

Os dados coletados foram obtidos durante um projeto de monitoramento a longo prazo da avifauna do parque. A atividade de captura das aves ocorreu entre julho de 2014 e julho de 2015. Em localidades selecionadas aleatoriamente, foram instaladas entre quatro e seis redes de neblina, que permaneciam abertas por até quatro horas, no período da manhã, e eram checadas a cada 30 minutos. As redes mediam $12 \mathrm{~m}$ por $2,5 \mathrm{~m}$ e apresentavam dois tipos de malhas: $30 \mathrm{~mm}$ e $36 \mathrm{~mm}$. O esforço amostral foi de 29 dias de captura e $20.880 \mathrm{~m}^{2} \mathrm{x}$ h. As aves capturadas foram medidas, pesadas, anilhadas e fotografadas, sendo liberadas no mesmo local de captura. Os sacos de contenção foram verificados quanto à presença de fezes contendo sementes de pau-incenso. O período de permanência das aves dentro dos sacos foi, em média, 5 minutos e correspondeu apenas ao tempo na qual estas aguardavam o manuseio para a obtenção de dados morfométricos. As sementes encontradas foram encaminhadas ao Laboratório de Sementes da Seção de Silvicultura do Instituto Florestal para a realização dos testes de germinação.

As sementes coletadas nas fezes foram imediatamente colocadas para germinar e comparadas com a amostra controle, constituída de sementes obtidas diretamente de frutos maduros iniciando o processo de abertura das cápsulas em agosto de 2014. O tratamento controle contou com quatro repetições com 25 sementes cada, provenientes de dez árvores distintas. Já as sementes obtidas nas fezes originaram seis repetições que apresentaram número variável de sementes. A diferença no tamanho das amostras ocorreu devido à dificuldade de obtenção das sementes nas fezes e à necessidade de se iniciar os testes rapidamente. 
Tanto as sementes coletadas nas fezes, como as da amostra controle foram semeadas sobre substrato de papel dentro de caixas acrílicas medindo $11 \times 11 \times 4 \mathrm{~cm}$, conhecidas como gerbox. Essas caixas foram colocadas dentro de germinadores, do tipo BOD, regulados para a temperatura de $25{ }^{\circ} \mathrm{C}$, com fotoperíodo de 8 horas de luz.

Para a determinação da assembleia de aves consumidoras dos frutos de pau-incenso, foram realizadas também 10 horas de observação focal de árvores com frutos maduros, obtidos durante as atividades de captura. O número de espécies consumidoras e o total de registros de alimentação (feeding bouts) foram comparados aos resultados obtidos em outros estudos com 10 horas de observação focal para seis espécies de arbóreas ornitocóricas ocorrentes no PEAL, três nativas e três exóticas (Fonseca e Antunes, 2007; Omote et al., 2014).
O teste qui-quadrado com correção de Yates foi efetuado para comparar as taxas de germinação entre os tratamentos. Utilizamos o programa BioEstat 5.0 (Ayres et al., 2010) e foi considerado significativo $\mathrm{p}<0,05$.

\section{RESULTADOS}

Foram capturados 102 indivíduos pertencentes a 12 espécies de aves conhecidas por incluírem frutos em suas dietas (Tabela 1). Destes, 84 eram representantes da família Turdidae, sendo que fezes com sementes de pau-incenso foram encontradas em oito indivíduos desta família. Esse valor representa, respectivamente, $7 \%$ e $10 \%$ do total de frugívoros e turdídeos capturados. Ao todo foram encontradas 107 sementes nas fezes, entre uma a 15 sementes por indivíduo.

Tabela 1. Aves frugívoras capturadas no Parque Estadual Alberto Löfgren, entre julho de 2014 e julho de 2015.

Table 1. Frugivorous birds captured in Alberto Löfgren State Park between July 2014 and July 2015.

\begin{tabular}{lcc}
\hline Táxon & $\begin{array}{c}\mathrm{N}^{\circ} \text { de indivíduos } \\
\text { capturados }\end{array}$ & $\begin{array}{c}\text { No de indivíduos que apresentaram } \\
\text { sementes nas fezes }\end{array}$ \\
\hline Ordem Columbiformes & 1 \\
Columbidae & 1 \\
Leptotila verreauxi Bonaparte, 1855 & \\
Leptotila rufaxilla (Richard \& Bernard, 1792) & \\
Ordem Passeriformes & 1 \\
Rhynchocyclidae & 3 \\
Mionectes rufiventris Cabanis, 1846 & 14 \\
Turdidae & 50 \\
Turdus flavipes Vieillot, 1818 & 3 \\
Turdus leucomelas Vieillot, 1818 & 14 \\
Turdus rufiventris Vieillot, 1818 & \\
Turdus amaurochalinus Cabanis, 1850 & 5 \\
Turdus albicollis Vieillot, 1818 & 1 \\
Thraupidae & 3 \\
Trichothraupis melanops (Vieillot, 1818) & \\
Tachyphonus coronatus (Vieillot, 1822) & \\
Coereba flaveola (Linnaeus, 1758) & 6 \\
Cardinalidae & \\
Habia rubica (Vieillot, 1817) &
\end{tabular}


No tratamento controle, a taxa de germinação variou entre 48 e $84 \%$ por repetição e, no total, $66 \%$ das sementes germinaram. No tratamento com o material obtido nas fezes das aves, foi possível efetuar seis repetições de oito a 31 sementes, referentes aos diferentes dias de amostragem. Neste tratamento, a taxa de germinação variou entre 13 e $81 \%$ por repetição e, no total, $47(44 \%)$ das sementes germinaram. Não houve diferença significativa nas taxas de germinação entre os tratamentos $\left(x^{2}=2,867\right.$ g.l. $\left.=1 \mathrm{p}=0,09\right)$.
Além desses turdídeos, em observações focais foram detectadas outras quatro espécies de aves consumindo frutos do pau-incenso: o jacuguaçu Penelope obscura Temminck, 1815, o pica-pau-de-cabeça-amarela Celeus flavescens (Gmelin, 1788), a maitaca-verde Pionus maximiliani (Kuhl, 1820) e o sabiá-branco Turdus leucomelas Vieillot, 1818. Quando comparada a outras espécies arbóreas zoocóricas ocorrentes no PEAL, observa-se que o pau-incenso apresenta menor riqueza de visitantes e menor número de feeding bouts (Tabela 2).

Tabela 2. Comparação da riqueza de aves e o total de feeding bouts, em 10 horas de observação, entre Pittosporum undulatum e outras seis espécies zoocóricas pesquisadas no PEAL. * indica espécie exótica.

Table 2. Comparison of bird richness and total of feeding bouts, in 10 hours of observation, between Pittosporum undulatum and other six zoochorous species researched in PEAL. * indicates exotic species.

\begin{tabular}{lccc}
\hline \multicolumn{1}{c}{ Espécie } & Riqueza de Visitantes & Total de Feeding Bouts & Referência \\
\hline Alchornea sidifolia Müll. Arg. & 21 & 218 & Fonseca e Antunes, 2007 \\
Eugenia uniflora L. * & 10 & 94 & Fonseca e Antunes, 2007 \\
Euterpe edulis Mart. & 10 & 116 & Omote et al. 2014 \\
Morus nigra L.* & 15 & 206 & Fonseca e Antunes, 2007 \\
Ocotea puberula (Rich.) Nees & 16 & 59 & Fonseca e Antunes, 2007 \\
Phoenix roebelenii O'Brien* & 19 & 236 & Omote et al., 2014 \\
Pittosporum undulatum Vent.* & 7 & 15 & Presente estudo \\
\hline
\end{tabular}

\section{DISCUSSÃO}

Os sabiás são abundantes na área de estudo,constituindo o principal grupo de dispersores de sementes (Cerisola et al., 2007; Fonseca e Antunes, 2007; Omote et al., 2014). Dentre as espécies de aves capturadas, as sementes de pau-incenso foram encontradas apenas nas fezes de três espécies da família Turdidae. Os sabiás são aves que apresentam o comportamento de "engolidores" de frutos (Parrini, 2015), o que pode ter facilitado o encontro das sementes de pau-incenso em suas fezes. 
A zoocoria contribui para a deposição das sementes longe da planta-mãe, o que pode favorecer sua germinação (Pjil, 1972). Apesar do limitado número de sementes obtidas em fezes e dospoucos feeding bouts observados, a interação entre o pau-incenso e os sabiás pode ter favorecido a dispersão dessa árvore na área de estudo. Em apoio a essa constatação, em áreas invadidas na Austrália, o principal consumidor das sementes de pau-incenso é Turdus merula Linnaeus, 1758, espécie exótica introduzida da Europa (Gleadow e Rowan, 1982).

Nossos resultados com o pau-incenso mostram que os efeitos da escarificação (retirada da camada que envolve as sementes), graças à passagem da semente pelo trato digestório das aves, não apresenta efeitos significativos sobre a germinação. Contudo, é sabido que os efeitos da escarificação na germinação variam entre as espécies vegetais e de acordo com a espécie animal dispersora, dependendo também, de uma série de características próprias das sementes individuais, como tamanho e espessura da casca (Barnea et al., 1991; Traveset, 1998).

A germinação das sementes também pode ser afetada por outros fatores envolvidos na frugivoria, como a remoção de inibidores de germinação presentes na polpa dos frutos e fertilização pelo material fecal que envolve as sementes (Traveset e Verdú, 2002). Assim, outra forma de avaliar a influência da ingestão das sementes de pau-incenso na taxa de germinação seria executar um estudo comparativo entre a germinação das sementes, que passaram pelo trato digestório das aves, e das sementes intactas ainda envoltas pela polpa dos frutos (Yagihashi et al., 1998; 1999; Robertson et al., 2006).Outra abordagem interessante avaliaria a influência da fertilização, comparando a taxa de germinação de sementes defecadas separadas das fezes e de sementes defecadas ainda envoltas em fezes.
O consumo das sementes pelos sabiás pode ser um fator que possibilitou o estabelecimento do pau-incenso em locais não favoráveis à ocorrência de hidrocoria. Isso permite, por exemplo, que essa árvore colonize áreas localizadas em altitudes mais elevadas do que o ponto inicial de seu plantio. Observamos esse padrão em relação ao contíguo Parque Estadual da Cantareira, em altitude em torno de $50 \mathrm{~m}$ acima do PEAL, e que apresenta trechos com diversos indivíduos da espécie estabelecidos (observação pessoal).

As quatro espécies de sabiás registradas consumindo frutos de pau-incenso apresentam diferenças quanto aos habitats utilizados, o que pode refletir no seu desempenho como dispersores.Um dos fatores mais importantes para o estabelecimento de plântulas de pau-incenso é o sombreamento (Gleadow e Rowan, 1982). O sabiá-coleira utiliza o interior do sub-bosque com mais frequência que as outras espécies de sabiás, o que poderia permitir a deposição dassementes de pau-incenso em sítios mais favoráveis ao seu desenvolvimento.

O consumo dos frutos do pau-incenso por aves foi baixo quando comparado aos dados obtidos para outras espécies vegetais ocorrentes no PEAL, o que pode indicar que os frutos de $P$. undulatum são menos atrativos para as aves frugívoras. Essa observação se corrobora pela afirmação de Mielke (2012) de que muitos herbívoros não apreciam as sementes resinosas do pau-incenso. Dentre as espécies observadas consumindo os frutos de P. undulatum, no PEAL, a maitaca apesar de ingerir a polpa, é principalmente uma predadora de sementes e provavelmente, não contribui significativamente para a dispersão do pau-incenso se comparada aos demais consumidores, que ingerem o fruto ou as sementes inteiras. Apesar de seu tamanho relativamente pequeno, as sementes de pau-incenso estão dentro do espectro que é predado por essa ave (Galetti, 1993). 
Concluímos que na Mata Atlântica do Sudeste brasileiro a interação entre o pau-incenso e aves frugívoras pode permitir a colonização de novas áreas por esta espécie exótica. Uma vez ocorrido esse estabelecimento, a dinâmica de ocupação pode prescindir da interação, devido às características biológicas do pauincenso, favoráveis à sua disseminação. Mais estudos são necessários para esclarecer a importância dos dispersores na germinação das sementes do pau-incenso. Esses dados são importantes para a compreensão dos processos que atuam na disseminação de espécies invasoras, como o P. undulatum, proporcionando melhores subsídios para as ações de manejo no interior de unidades de conservação.

\section{AGRADECIMENTOS}

Agradecemos ao Instituto Florestal por meio da COTEC (Processo SMA 260108008.500/2009) e à gestora do Parque Estadual Alberto Löfgren, Roselaine Barros Machado, pela autorização para o desenvolvimento do projeto. À pesquisadora Marina Mitsue Kanashiro pelo auxílio na confecção da Figura 1. Para as atividades de captura e manuseio, contouse com as autorizações SISBIO para captura (autorização 29073-4) e CEMAVE para anilhamento (registro 581572, autorização 1256/7). Somos gratos aos dois revisores anônimos pelas sugestões e críticas pertinentes, que aprimoraram o manuscrito.

\section{REFERÊNCIAS BIBLIOGRÁFICAS}

AYRES, M. et al. BioEstat 5.0 - aplicações estatísticas nas áreas das ciências biológicas e médicas. Brasília, DF: CNPq, 2010. 290 p.
BARNEA, A.; YOM-TOV, Y.; FRIEDMAN, J. Does ingestion by birds affect seed germination? Functional Ecology, v. 5, p. 394-402, 1991.

BLUM, C.T. et al. Espécies vegetais invasoras em comunidades florestais nativas nas margens da represa do Vossoroca, APA de Guaratuba, Paraná, Brasil. In: SIMPÓSIO BRASILEIRO SOBRE ESPÉCIES EXÓTICAS INVASORAS, 2005, Brasília, DF. Anais... Brasília, DF: Ministério do Meio Ambiente, 2005. 14 p.

CERISOLA, C.M.; ANTUNES, A.Z.; PORTCARVALHO, M. Consumo de frutos de Euterpe edulis Martius (Arecaceae) por vertebrados no Parque Estadual Alberto Löfgren, São Paulo, Sudeste do Brasil. IF Série Registros, n. 31, p. 167 71, 2007.

COOPER, R.P. Birds feeding on Pittosporum seeds. Emu, v. 59, p. 60-1, 1959.

D'AVILA, G. et al. The role of avian frugivores on germination and potential seed dispersal of the Brazilian Pepper Schinus terebinthifolius. Biota Neotropica, v. 10, p. 45-51, 2010.

FONSECA, F.; ANTUNES, A. Frugivoria e predação de sementes por aves no Parque Estadual Alberto Löfgren, São Paulo, SP. Revista do Instituto Florestal, v. 19, p. 81-91, 2007.

GALETTI, M. Diet of scaly-headed parrot (Pionus maximiliani) in a semi-deciduous forest in southeastern Brazil. Biotropica, v. 25, p. 419-425, 1993.

GLEADOW, R.M.; ROWAN, K.S. Invasion by Pittosporum undulatum of the forests of Central Victoria: effects of temperature and light on growth and drought resistance. Australian Journal of Botany, v. 30, p. 347-57, 1982. 
GOODLAND, T.; HEALEY, J.R. The invasion of Jamaican montane rainforests by the Australian tree Pittosporum undulatum. Bangor: School of Agricultural and Forest Sciences, University of Wales, 1996. 55 p.

. The effect of Pittosporum undulatum on the native vegetation of the Blue Mountains of Jamaica. Bangor: School of Agricultural and Forest Sciences, University of Wales, 1997. $41 \mathrm{p}$.

HELENO, R.H. et al. The role of avian 'seed predators' as seed dispersers. Ibis, v. 153, p. 199-203, 2011.

INSTITUTO HÓRUS. Pittosporum undulatum. 2016. Disponível em: <http://www.institutohorus.org.br>. Acesso em: 1 mar. 2016.

MIELKE, E.C. Árvores exóticas invasoras em Unidades de Conservação de Curitiba, Paraná: subsídios ao manejo e controle. 2012. $103 \mathrm{f}$. Dissertação (Mestrado em Agronomia) Universidade Federal do Paraná, Curitiba.

et al. Espécies exóticas invasoras arbóreas no Parque da Barreirinha em Curitiba: registro e implicações. Ciência Florestal, v. 25, p. $327-336,2015$.

MOERMOND, T.C.; DENSLOW, J.S. Fruit choice in neotropical birds: effects of fruit type and accessibility on selectivity. Journal of Animal Ecology, v. 52, p. 407-420, 1983.

MULLETT, T.L. Some characteristics of a native environmental weed: Pittosporum undulatum. In: BISHOP, A.C. et al. AUSTRALIAN WEEDS CONFERENCE, 12., 1999, Hobart. Proceedings... Devonport: Tasmanian Weed Society, 1999a. p. 592-595.

_. Ecological aspects of sweet pittosporum (Pittosporum undulatum Vent.): implications for control and management. In: BISHOP, A.C. et al. MULLETT, T.L. AUSTRALIAN WEEDS CONFERENCE, 12. 1999, Hobart. Proceedings... Devonport: Tasmanian Weed Society, 1999 Proceedings... Devonport: Tasmanian Weed Society, 1999b. p. 489-492.
OMOTE, T.; ANTUNES, A.Z:; MATSUKUMA, C.K. Comparação de aspectos fenológicos e de frugivoria entre a palmeira nativa Euterpe edulis Mart. e a palmeira exótica Phoenix roebelenii O'Brien (Arecaceae) no Sudeste do Brasil. Revista do Instituto Florestal, v. 26, p. 169-181, 2014.

PARRINI, R. Quatro estações: História Natural das aves na Mata Atlântica - uma abordagem trófica. Rio de Janeiro: Technical Books, 2015. 354 p.

PIJL, L. van der. Principles of dispersal in higher plants. New York: Springer Verlag, 1972. $162 \mathrm{p}$.

ROBERTSON, A.W. et al. Assessing the benefits of frugivory for seed germination: the importance of the deinhibition effect. Functional Ecology, v. 20, p. 58-66, 2006.

ROSE, S. Influence of suburban edges on invasion of Pittosporum undulatum into the bushland of northern Sydney, Australia. Australian Journal of Ecology, v. 22, p. 89-99, 1997.

SANTANA, O.A.; ENCINAS, J.I. Levantamento das espécies exóticas arbóreas e seu impacto nas espécies nativas em áreas adjacentes a depósitos de resíduos domiciliares. Biotemas, v. 21, p. $29-38,2008$.

SÃO PAULO (Estado). Fundação Florestal. Parque Estadual da Cantareira: plano de manejo. São Paulo, 2009. 541 p.

Fundação Florestal. Parque Estadual da Alberto Löfgren: Plano de Manejo. São Paulo, 2012. 710 p.

TRAVESET, A. Effect of seed passage through vertebrate frugivores' guts on germination: a review. Perspectives in Plant Ecology, Evolution and Systematics, v. 1/2, p. 151-190, 1998.

.; VERDÚ, M. A Meta-analysis of the effect of gut treatment on seed germination. $\mathbf{C A B}$ International, p. 339-350, 2002. 
CAMPAGNOLI, M.L. et al. Aves e a disseminação do pau-incenso.

YAGIHASHI, T:; HAYASHIDA, M.; MYIAMOTO, T. Effects of bird ingestion on seed germination of Sorbus commixta. Oecologia, v. 114, p. 209-212, 1998.

. Effects of bird ingestion on seed germination of two Prunus species with different fruit-ripening seasons. Ecological Research, v. 14, p. 71-76, 1999. 